\title{
Fascial plane blocks: anatomical structures that affect the spread of local anesthetic
}

\author{
Kunihisa Hotta ${ }^{1}[$ ] \\ Received: 8 April 2019 / Accepted: 24 April 2019 / Published online: 9 May 2019 \\ (c) Japanese Society of Anesthesiologists 2019
}

Keywords Fascial plane block · Ultrasound · Local anesthetic $\cdot$ Tendinous septum

The endpoint of a peripheral nerve block procedure is the deposition of local anesthetic around a peripheral nerve, causing a nerve conduction blockade. When performing ultrasound-guided sciatic nerve block, the needle is closely adjusted to the sciatic nerve using the ultrasound image, and then the local anesthetic is administered. The low echo of the local anesthetic surrounds the high echo of the sciatic nerve, and this image is called the "donut sign." This endpoint of the ultrasound-guided procedure appears to be more reasonable to anesthesiologists compared with paresthesia and motor response by nerve stimulation.

In recent years, several articles evaluating new ultrasound-guided fascial plane blocks have been published because of the advancement in the ultrasound-guided block procedure and knowledge of the musculoskeletal ultrasound anatomy [1]. In addition, ultrasound guidance is advantageous in performing fascial plane blocks, and the correct fascial plane to which local anesthetic should be administered is determined using the ultrasound image. Conventional fascial click was reported to be an unreliable endpoint for fascial plane blocks [2]. In addition, fascial plane blocks are considered at low risk of nerve injury and a relatively easy technique. The endpoint of the ultrasound-guided procedure is the spread of local anesthetic on the target fascial plane; thus, a donut sign is not required. However, the whole spread of local anesthetic on the fascial plane using a 2D ultrasound image is rarely confirmed in our clinical practice. The use of a large amount of local anesthetic may make the analgesic effect more reliable. On the contrary, it increases the risk of systemic toxicity and unintentional motor block.

Kunihisa Hotta

hottak@jichi.ac.jp

1 Department of Anesthesiology and Critical Care Medicine, Jichi Medical University School of Medicine, 3311-1 Yakushiji, Shimotsuke-shi, Tochigi 329-0498, Japan
For example, although the adductor canal block is considered to preserve the motor function of the quadratus femoris muscle [3], motor weakness after adductor canal block has been reported [4]. Recent anatomical studies suggest that "femoral triangle block" is a more accurate description of the neurovascular compartment block at the level of the midthigh compared with "adductor canal block". Moreover, the quadratus lumborum (QL) block is a unique fascial plane block, which was originally used for abdominal surgeries. Since local anesthetic can spread to the psoas major and iliacus muscles via the transversalis fascia overlying the QL muscle, the QL block may result in the lumbar plexus block. The spread of injectate following transmuscular QL block in cadavers stained the upper branches of the lumbar plexus and psoas major muscle [5]. Unexpected motor weakness in the lower extremity occurred following lateral QL block with $20 \mathrm{ml}$ of levobupivacaine $0.25 \%$ in a patient undergoing laparoscopic endometriosis excision [6]. The potential risk of quadriceps weakness should be considered when performing QL block for abdominal surgery. Thus, understanding the spread of local anesthetic on the fascial plane and administering the appropriate local anesthetic dose contribute to improving the safety and efficacy of the fascial plane blocks.

In three articles that were recently published in the Journal of Anesthesia, Ohgoshi and the colleagues have examined the effect of the fascial plane blocks in the posterior neck region and identified the anatomical structure limiting the spread of local anesthetics [7-9]. In their cadaver study, the dyes were injected into the inter-semispinal plane (ISP block) and medial head of the semispinalis capitis muscle [greater and third occipital nerve (GTO) block] [7]. They found that the dyes were clearly separated by the tendinous septum in the semispinalis capitis muscle. This finding is the answer to their clinical question as to why the sensory block areas obtained by the ISP and GTO blocks did not overlap. In their previous volunteer study, they measured 
the sensory block area following the ISP block. The ISP block using $20 \mathrm{~mL}$ of local anesthetic produced the sensory block area innervated by the dorsal ramus of $\mathrm{C} 4$ to $\mathrm{T} 4$, but no effect was observed on the GTOs [8]. Moreover, in their other study, they reported a simple ultrasound-guided block procedure named GTO block [9]. This procedure simultaneously blocked both the GTOs with a single local anesthetic injection into the medial head of the semispinalis capitis muscle at the level of the first cervical vertebra. The explanation based on the anatomical basis of this finding was that both nerves were in close proximity at this site in the semispinalis capitis muscle. Lastly, Ohgoshi et al. found that the tendinous septum of the semispinalis capitis muscle had a relatively strong connection with the dorsal edge of the semispinalis cervicis muscle, and this structure significantly affected the spread of injectate [7].

Some fascial plane blocks, such as the transversus abdominis plane and rectus sheath blocks, are widely used for patients undergoing abdominal surgery as standard analgesic techniques in our daily clinical practice [10]. The advance of the ultrasound-guided block procedure and knowledge of musculoskeletal ultrasound anatomy contribute to the invention of the new block techniques, and they are of great interest in the field of regional anesthesia. However, controversies regarding the spread of local anesthetics and the optimum doses in various kinds of fascial plane blocks are still noted. Studies evaluating anatomical structures that affect the clinical outcomes of the fascial plane blocks are of great importance. More clinical trials are required for the improvement of our clinical practice.
2. Weintraud M, Marhofer P, Bösenberg A, Kapral S, Willschke H, Felfernig M, Kettner S. Ilioinguinal/iliohypogastric blocks in children: where do we administer the local anesthetic without direct visualization? Anesth Analg. 2008;106:89-93.

3. Zhao XQ, Jiang N, Yuan FF, Wang L, Yu B. The comparison of adductor canal block with femoral nerve block following total knee arthroplasty: a systematic review with meta-analysis. J Anesth. 2016;30:745-54.

4. Chen J, Lesser JB, Hadzic A, Reiss W, Resta-Flarer F. Adductor canal block can result in motor block of the quadriceps muscle. Reg Anesth Pain Med. 2014;39:170-1.

5. Adhikary SD, El-Boghdadly K, Nasralah Z, Sarwani N, Nixon $\mathrm{AM}$, Chin KJ. A radiologic and anatomic assessment of injectate spread following transmuscular quadratus lumborum block in cadavers. Anaesthesia. 2017;72:73-9.

6. Wikner M. Unexpected motor weakness following quadratus lumborum block for gynaecological laparoscopy. Anaesthesia. 2017;72:230-2.

7. Ohgoshi Y, Usui Y, Ishikawa S, Takeda Y, Ohtsuka A. The tendinous septum of the semispinalis capitis muscle spatially separates the dorsal ramus between C3 and C4. J Anesth. 2018;32:774-6.

8. Ohgoshi Y, Nishizakura R, Takahashi Y, Takeda K, Nakayama H, Kawamata M, Kurahashi K. Novel ultrasound-guided intersemispinal plane block: a comparative pilot study in healthy volunteers. J Anesth. 2018;32:143-6.

9. Kariya K, Usui Y, Higashi N, Nakamoto T, Shimbori H, Terada S, Takahashi H, Ueta H, Kitazawa Y, Sawanobori Y, Okuda Y, Matsuno K. Anatomical basis for simultaneous block of greater and third occipital nerves, with an ultrasound-guided technique. J Anesth. 2018;32:483-92.

10. Ma N, Duncan JK, Scarfe AJ, Schuhmann S, Cameron AL. Clinical safety and effectiveness of transversus abdominis plane (TAP) block in post-operative analgesia: a systematic review and metaanalysis. J Anesth. 2017;31:432-52.

Publisher's Note Springer Nature remains neutral with regard to jurisdictional claims in published maps and institutional affiliations.

\section{References}

1. Yamauchi M, Sato Y. Complex issues in new ultrasound-guided nerve blocks: how to name, where to inject, and how to publish. J Anesth. 2018;32:283-7. 